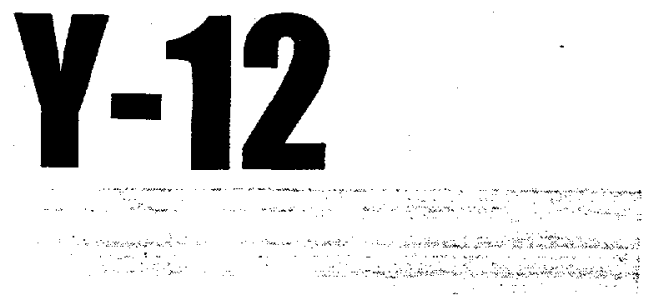

\section{OAK RIDGE} $\mathrm{Y}-12$ PLANT

LOCKHEED MATTIN
Revised Emergency Planning and Community Right-to-Know Act (EPCRA), Section 313, Toxic Chemical Release Reporting for Calendar Year 1998

R. A. Evans

Environment, Safety, and Health Organization
Y-12 Plant

LOCKHEED MARTIN ENERGY SYSTEMS, INC.

managed by
Lockheed Martin Energy Systems, Inc. US DEPART for the U.S. DEPARTMENT OF ENERGY Under Contract No. DE-AC05-84-OR21400 


\section{DISCLAIMER}

This report was prepared as an account of work sponsored by an agency of the United States Government. Neither the United States Government nor any agency thereof, nor any of their employees, makes any warranty, express or implied, or assumes any legal liability or responsibility for the accuracy, completeness, or usefulness of any information, apparatus, product, or process disclosed, or represents that its use would not infringe privately owned rights. Reference herein to any specific commerciai product, process, or service by trade name, trademark, manufacturer, or otherwise, does not necessarily constitute or imply its endorsement, recommendation, or favoring by the United States Government or any agency thereof. The views and opinions of authors expressed herein do not necessarily state or reflect those of the United States Government or any agency thereof. 


\section{DISCLAIMER}

Portions of this document may be illegible in electronic image products. Images are produced from the best available original document. 


\title{
Revised Emergency Planning and Community Right-to-Know Act (EPCRA), Section 313, Toxic Chemical Release Reporting for Calendar Year 1998
}

\section{January 2000}

\author{
R. A. Evans \\ Environment, Safety, and Health Organization \\ Y-12 Plant
}

\author{
Prepared by the \\ Oak Rdge Y-12 Plant \\ Oak Ridge, Tennessee 37831 \\ managed by \\ Lockheed Martin Energy Systems, Inc. \\ for the \\ U.S. DEPARTMENT OF ENERGY \\ Under Contract No. DE-ACO5-84-OR21400
}


United States

Environmental Protection

Section 313 of the Emergency Planning and Community Right-to-Know Act of 1986 , Agency

also known as Title III of the Superfund Amendments and Reauthorization Act

TOXIC CHEMICAL RELEASE

INVENTORY REPORTING FORM

WHERE TO SEND COMPLETED FORMS: 1 . EPCRA Reporting Center P.O Box 3348

Merrifield. VA 22116-3348

ATTN: TOXIC CHEMICAL RELEASE INVENTORY
2. APPROPRIATE STATE OFFICE (See instructions in Appendix F)

\begin{tabular}{|l|l|}
\hline $\begin{array}{l}\text { Enter " } \mathrm{X} \text { " here if this } \\
\text { is a revision }\end{array}$ \\
\hline For EPA use only \\
\hline
\end{tabular}

Important: See instructions to determine when "Not Applicable (NA)" boxes should be checked.

\section{PART I. FACILITY IDENTIFICATION INFORMATION}

\section{SECTION 1. REPORTING YEAR 1998}

\section{SECTION 2. TRADE SECRET INFORMATION}

\begin{tabular}{|c|c|c|c|c|c|c|c|c|}
\hline \multirow[b]{2}{*}{2.1} & \multicolumn{3}{|c|}{ Are you claiming the toxic chemical identified on page 3 trade secret? } & \multirow[b]{2}{*}{2.2} & \multirow{2}{*}{ Is this copy } & \multirow{2}{*}{ Sanitized } & . & \multirow{2}{*}{ Unsanitized } \\
\hline & $\begin{array}{l}\text { Yes (Answer question } 2.2 \text {; } \\
\text { Attach substantiation forms) }\end{array}$ & $x$ & $\begin{array}{c}\text { No (Do not answer 2.2; } \\
\text { Go to Section 3) }\end{array}$ & & & & & \\
\hline
\end{tabular}

SECTION 3. CERTIFICATION (Important: Read and sign after completing all form sections.)

I hereby certify that I have reviewed the attached documents and that, to the best of my knowledge and belief, the submitted information is true and complete and that the amounts and values in this report are accurate based on reasonable estimates using data available to the preparers of this report.

\begin{tabular}{|l|l|l|}
\hline Name and official title of owner/operator or senior management official: & Signature: & Date Sigrea: \\
\hline R. D. DEMPSEY & ACTING DIRECTOR, TECHNICAL SUPPORT OMISION & \\
\hline
\end{tabular}

\section{SECTION 4. FACILITY IDENTIFICATION}

\begin{tabular}{|c|c|c|c|}
\hline 4.1 & & TRI Faciiity ID Number & 37a31-SOKRD-BEARC \\
\hline Facility or Estabtishment Name & & \multicolumn{2}{|c|}{ Facility or Establishment Name or Mailing Address (if different from street acdress) } \\
\hline \multicolumn{2}{|l|}{ U.S. DOE Y.12 PLANT } & & \\
\hline Street & & Mailing Address & \\
\hline GEAR CREEK ROAD & & POST OFFICE BOX 2001 & \\
\hline City/CountyiStaterZip Code & \multirow[b]{2}{*}{ TN 37831-2001 } & City/County/State/Zip Code & \\
\hline OAKRIOGE & & OAKRIDGE & TN $\quad 37831-2 \mathrm{CO}$ \\
\hline
\end{tabular}

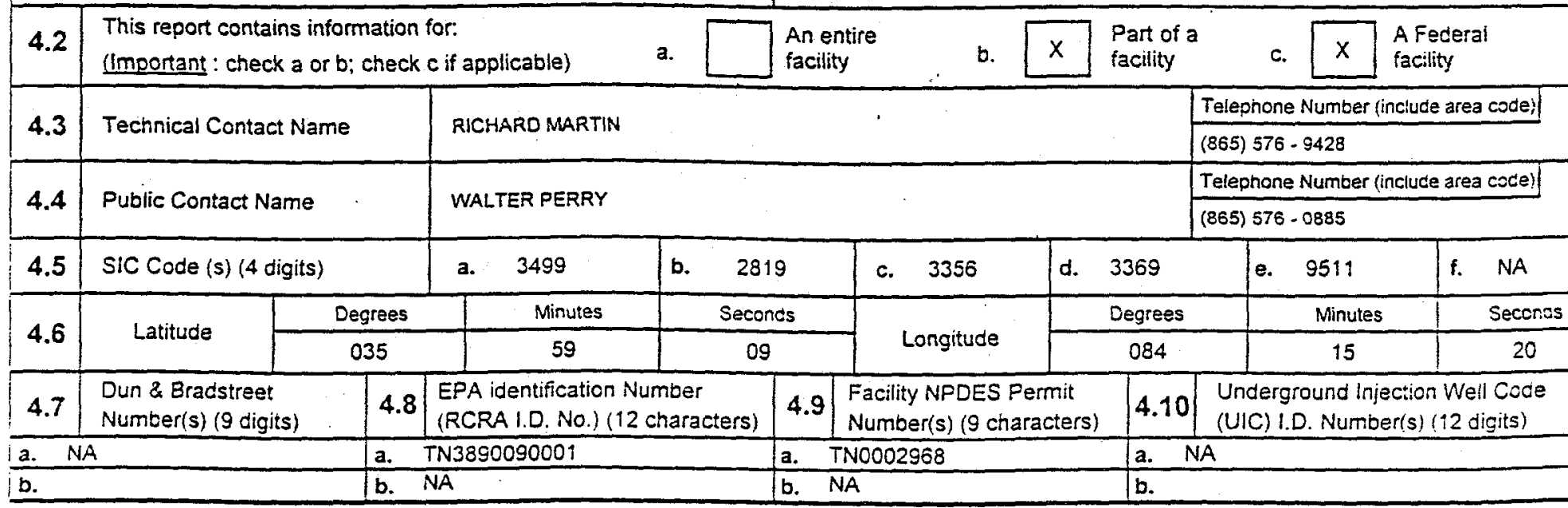

SECTION 5. PARENT COMPANY INFORMATION

\begin{tabular}{|l|l|l|l|l|l|}
\hline 5.1 & Name of Parent Company & NA & & \multicolumn{1}{|c|}{ U.S. DEPARTMENT OF ENERGY } \\
\hline 5.2 & Parent Company's Dun \& Bradstreet Number & NA & $\mathrm{X}$ & \\
\hline
\end{tabular}




\section{EPA FORM R \\ PART II. CHEMICAL-SPECIFIC INFORMATION}

\begin{tabular}{|l|}
\hline TRI Faclity ID Numcer \\
\hline 37331 -SOKRD-BEARC \\
\hline TOxic Chemical. Category or Genenc Name \\
\hline LEAO
\end{tabular}

\section{SECTION 1. TOXIC CHEMICAL IDENTITY}

(Important: DO NOT complete this section if you completed Section 2 below.)

\begin{tabular}{|c|c|}
\hline \multirow{2}{*}{1.1} & CAS Number (Important: Enter oniy one number exactly as it appears on the Section 313 list. Enter \\
\hline & 007439921 \\
\hline & Toxic Chemical or Chemical Category Name (Important: Enter only one name exactly as it appears on the Section 313 list.) \\
\hline & LEAD \\
\hline 1.3 & Generic Chemical Name (Important: Complete only if Part 1. Section 2.1 is checked "yes". Generic Name must be structurally descriptive) \\
\hline
\end{tabular}

SECTION 2. MIXTURE COMPONENT IDENTITY (Important: DO NOT complete this section if you completed Section 1 above.)

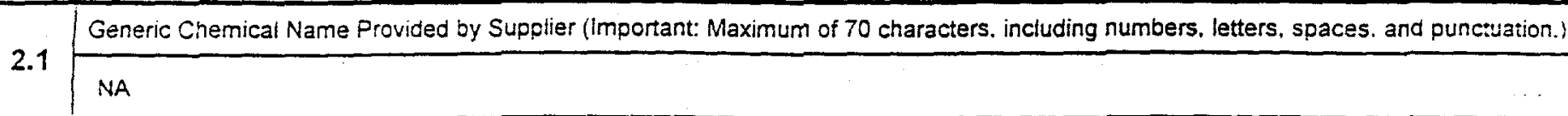

\section{SECTION 3. ACTIVITIES AND USES OF THE TOXIC CHEMICAL AT THE FACILITY} (Important: Check all that apply.)

\begin{tabular}{|c|c|c|c|c|c|c|c|c|}
\hline 3.1 & \multicolumn{3}{|c|}{ Manufacture the toxic chemical: } & 3.2 & Process the toxic chemical: & 3.3 & \multicolumn{2}{|c|}{ Otherwise use the toxic cher } \\
\hline a. & Produce & b. & Import & & & & & \\
\hline & \multicolumn{3}{|c|}{ If produce or impor: } & & & & & \\
\hline c. & \multicolumn{3}{|c|}{ For on-site useiprocessing } & a. & As a reactant & a. & & As a chemical processing aid \\
\hline d. & \multicolumn{3}{|c|}{ For sale/distribution } & b. & As a formulation component & b. & & As a manufacturing aid \\
\hline e. & \multicolumn{3}{|c|}{ As a byproduct } & c. & As an article component & c. & $x$ & Ancillary or other use \\
\hline f. & \multicolumn{3}{|c|}{ As an impurity } & d. & Repackaging & & & \\
\hline
\end{tabular}

SECTION 4. MAXIMUM AMOUNT OF THE TOXIC CHEMICAL ONSITE AT ANY TIME DURING THE CALENDAR YEAR

4.1 03 (Enter two-digit code from instruction package.)

SECTION 5. QUANTITY OF THE TOXIC CHEMICAL ENTERING EACH ENVIRONMENTAL MEDIUM ONSITE

\begin{tabular}{|c|c|c|c|c|c|c|c|}
\hline & & & & $\begin{array}{l}\text { A. Total Release (pounds/year) } \\
\text { (Enter range code or estimate") }\end{array}$ & $\begin{array}{l}\text { 8. Basis of estin } \\
\text { (enter code) }\end{array}$ & nate & C. \% From Stormwater \\
\hline 5.1 & $\begin{array}{l}\text { Fugitive or non-point } \\
\text { air emissions }\end{array}$ & NA & & 1 & 0 & & \\
\hline 5.2 & $\begin{array}{l}\text { Stack or point } \\
\text { air emissions }\end{array}$ & NA & & 10 & $E$ & & \\
\hline 5.3 & \multicolumn{3}{|c|}{$\begin{array}{l}\text { Discharges to receiving streams or } \\
\text { water bodies (enter one name per box) }\end{array}$} & & & & \\
\hline & \multicolumn{3}{|c|}{ Stream or Water Body Name } & & & & \\
\hline 5.3 .1 & \multicolumn{3}{|l|}{ EAST FORK POPLAR CREEK } & 840 & \multicolumn{2}{|l|}{$M$} & 000.00 \\
\hline \multicolumn{8}{|l|}{5.3 .2} \\
\hline \multicolumn{8}{|l|}{5.3 .3} \\
\hline 5.4 .1 & $\begin{array}{l}\text { Uncerground injection onsite } \\
\text { to Class I Wells }\end{array}$ & NA & $x$ & NA & & & \\
\hline 5.4 .2 & $\begin{array}{l}\text { Underground injection onsite } \\
\text { io Class } 1-V \text { Weils }\end{array}$ & NA & $x$ & NA & & & \\
\hline \multirow{2}{*}{\multicolumn{7}{|c|}{$\begin{array}{l}\text { If additional pages of Part II, Section } 5.3 \text { are attached, incicate the total number of pages in this box } \\
\text { and indicate the Part } 11 \text { Section } 5.3 \text { page number in this box. } 1 \text { (example: } 1,2,3 \text { etc) }\end{array}$}} & \\
\hline & & & & & & & \\
\hline
\end{tabular}


EPA FORM R

PART II. CHEMICAL - SPECIFIC INFORMATION (CONTINUED)
TRI Facility ID Number

37831-SDKRD-BEARC

Toxic Chemical. Category. or Generic Name LEAD

SECTION 5. QUANTITY OF THE TOXIC CHEMICAL ENTERING EACH ENVIRONMENTAL MEDIUM ONSITE (ContinuEd)

\begin{tabular}{|l|l|l|l|l|l|}
\hline \multicolumn{2}{|l|}{} & NA & $\begin{array}{r}\text { A. Total Release (pounds/year) (enter range } \\
\text { code* or estimate) }\end{array}$ & $\begin{array}{l}\text { B. Basis of Estimate } \\
\text { (enter coce) }\end{array}$ \\
\hline 5.5 & Disposal to land onsite & & & \\
\hline $5.5 .1 \mathrm{~A}$ & RCRA Subtitle C landfills & $\mathrm{X}$ & NA & 0 \\
\hline $5.5 .1 \mathrm{~B}$ & Other landfills & & 320 & \\
\hline 5.5 .2 & $\begin{array}{l}\text { Land treatment/appication } \\
\text { farming }\end{array}$ & $\mathrm{X}$ & NA & \\
\hline 5.5 .3 & Surface impoundment & $\mathrm{X}$ & NA & \\
\hline 5.5 .4 & Other disposal & & 0 & \\
\hline
\end{tabular}

SECTION 6. TRANSFERS OF THE TOXIC CHEMICAL IN WASTES TO OFF-SITE LOCATIONS

\subsection{DISCHARGES TO PUBLICLY OWNED TREATMENT WORKS (POTWS)}

\subsection{A Total Quantity Transferred to POTWs and Basis of Estimate}

\begin{tabular}{|l|c}
$\begin{array}{c}\text { 6.1.A.1. Total Transfers (pounds/year) } \\
\text { (enter range code* or estimate) }\end{array}$ & $\begin{array}{c}\text { 6.1.A.2 Basis of Estimate } \\
\text { (enter code) }\end{array}$ \\
\hline 7 & $\mathrm{M}$ \\
\hline
\end{tabular}

\begin{tabular}{|l|l|l|l|l|l|l|l|l|}
\hline 6.1.B. 1 & POTW Name & OEPARTMENT OF PUBLIC WORKS & & & \\
\hline POTW Address & POST OFFICE BOX 1 & & & & \\
\hline City & OAK RIDGE & & State & TN & County & ANDERSON & 37830. \\
\hline
\end{tabular}

\begin{tabular}{|c|c|c|c|c|}
\hline \multicolumn{2}{|c|}{$\begin{array}{ll}6.1 . B .2 & \text { POTW Name } \\
\end{array}$} & & & \\
\hline \multicolumn{2}{|c|}{ POTW Address } & . & & \\
\hline City & & State & County & $Z i p$ \\
\hline
\end{tabular}

If additional pages of Part II, Section 6.1 are attached, indicate the total number of pages

in this box 1 and indicate the Part II, Section 6.1 page number in this box $\square 1$ (example: $1,2,3$, etc.)

\section{SECTION 6.2 TRANSFERS TO OTHER OFF-SITE LOCATIONS}

\subsection{Off-Site EPA Identification Number (RCRA ID No.)}

Off-Site Lccation Name SAFETY-KLEEN (DEER PARK), INC.

\section{Off-Site Address 2027 BATTLEGROUND ROAD}

\begin{tabular}{|l|l|l|l|l|}
\hline City & DEER PARK & State & $\Gamma X$ & County \\
\hline
\end{tabular}

Is location under control of reporting faciilty or parent company? 


\section{EPA FORM R}

PART \| CHEMICAL-SPECIFIC INFORMATION.(CONTINUED)
TRI Facility ID Number

37831-SCKRD-BEARC

Toxic Chemical. Category or Generic Varie LEAD

\section{SECTION 6.2 TRANSFERS TO OTHER OFF-SITE LOCATIONS (Continued)}
A. Total Transfers (pounds/year)
(enter range code' or estimate)
B. Basis of Estimate
(enter code)

\begin{tabular}{|l|l}
\hline 1. 400 & 1 \\
\hline 2. & \\
\hline 3. & 3. \\
\hline 4. & 4 \\
\hline
\end{tabular}

\begin{tabular}{|l|l|}
\hline 1.0 & $1 . \mathrm{M72}$ \\
\hline 2. & 2. \\
\hline 3. & 3. \\
\hline 4. & 4. \\
\hline
\end{tabular}

NCD000648451

6.22 Off-Site EPA Identification Number (RCRA ID No.)

C. Type of Waste Treatment/Disposall

Recycling/Energy Recovery (enter code)

\begin{tabular}{l|l} 
Off-Site location Name & SAFETY-KLEEN (TS), INC.
\end{tabular}

Off-Site Address 208 WATLINGTON INDUSTRIAL DR.

\begin{tabular}{l|l|l|l|l|}
\hline City & REIDSVILLE & State & NC & County \\
\hline
\end{tabular}

Is location under control of reporting facility or parent company?

\begin{tabular}{|l|l|l|}
\hline $\begin{array}{c}\text { A. Total Transfers (pounds/year) } \\
\text { (enter range code* or estimate) }\end{array}$ & \multicolumn{1}{|c|}{$\begin{array}{c}\text { B. Basis of Estimate } \\
\text { (enter code) }\end{array}$} & $\begin{array}{c}\text { C. Type of Waste Treatment'Disposail } \\
\text { Recycling/Energy Recovery (enter code) }\end{array}$ \\
\hline $1 . \quad 960$ & 1.0 & $1 . \quad M 72$ \\
\hline 2. & 2. & 2. \\
\hline 3. & 3. & 3. \\
\hline 4. & 4. & 4. \\
\hline
\end{tabular}

SECTION 7A. ON-SITE WASTE TREATMENT METHODS AND EFFICIENCY

$X$ Not Applicable (NA) - Check here if no on-site waste treatment is applied to any

waste stream containing the toxic chemical or chemical category.

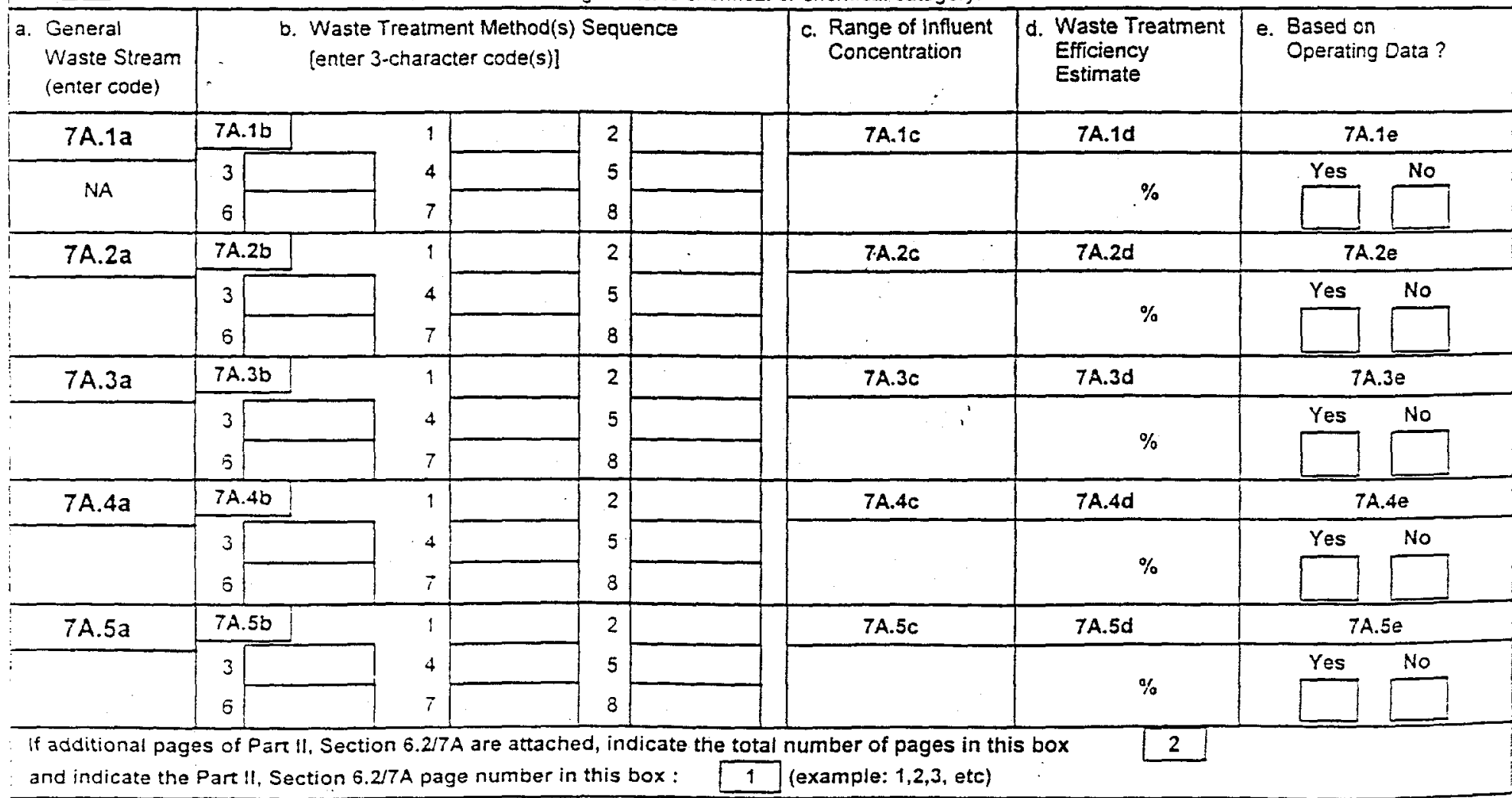




\section{EPA.FORM R}

\section{PART II CHEMICAL-SPECIFIC INFORMATION (CONTINUED)}

TRI Facility ID Number 37831-SDKRD-BEARC

Toxic Chemical. Category or Generic Name LEAD

\section{SECTION 6.2 TRANSFERS TO OTHER OFF-SITE LOCATIONS (Continued)}
A. Total Transfers (pounds/year)
B. Basis of Estimate
(enter range code" or estimate)
(enter code)
C. Type of Waste Treatmentrisposall
Recycling/Energy Recovery (enter code)

\begin{tabular}{|l|l}
\hline 1. & 1 \\
\hline 2. & 2 \\
\hline 3. & 3 \\
\hline 4. & 4 \\
\hline
\end{tabular}

\begin{tabular}{|l|l|}
\hline 1. & 1. \\
\hline 2. & 2. \\
\hline 3. & 3. \\
\hline 4. & 4. \\
\hline
\end{tabular}

6.23 Off-Site EPA Identification Number (RCRA ID No.)

SCD070375985

\begin{tabular}{l|l} 
Off-Site lecation Name & SAFETY-KLEEN (PINEWOOD), INC.
\end{tabular}

Off-Site Address ROUTE 1, BOX 255
City PINEWOOD
\begin{tabular}{|l|l|l|} 
State & SC & County \\
\hline
\end{tabular}

Is location under control of reporting facility or parent company?

A. Total Transfers (pounds/year) (enter range code* or estimate)

\begin{tabular}{|l|l}
\hline 1.6569 & 1. \\
\hline 2. & 2 \\
\hline 3. & 3 \\
\hline 4. & 4 \\
\hline
\end{tabular}

B. Basis of Estimate (enter code)

\section{SECTION 7A. ON-SITE WASTE TREATMENT METHODS AND EFFICIENCY}

Not Applicable (NA) - Check here if no on-site waste treatment is applied to any

waste stream containing the toxic chemical or chemical category.

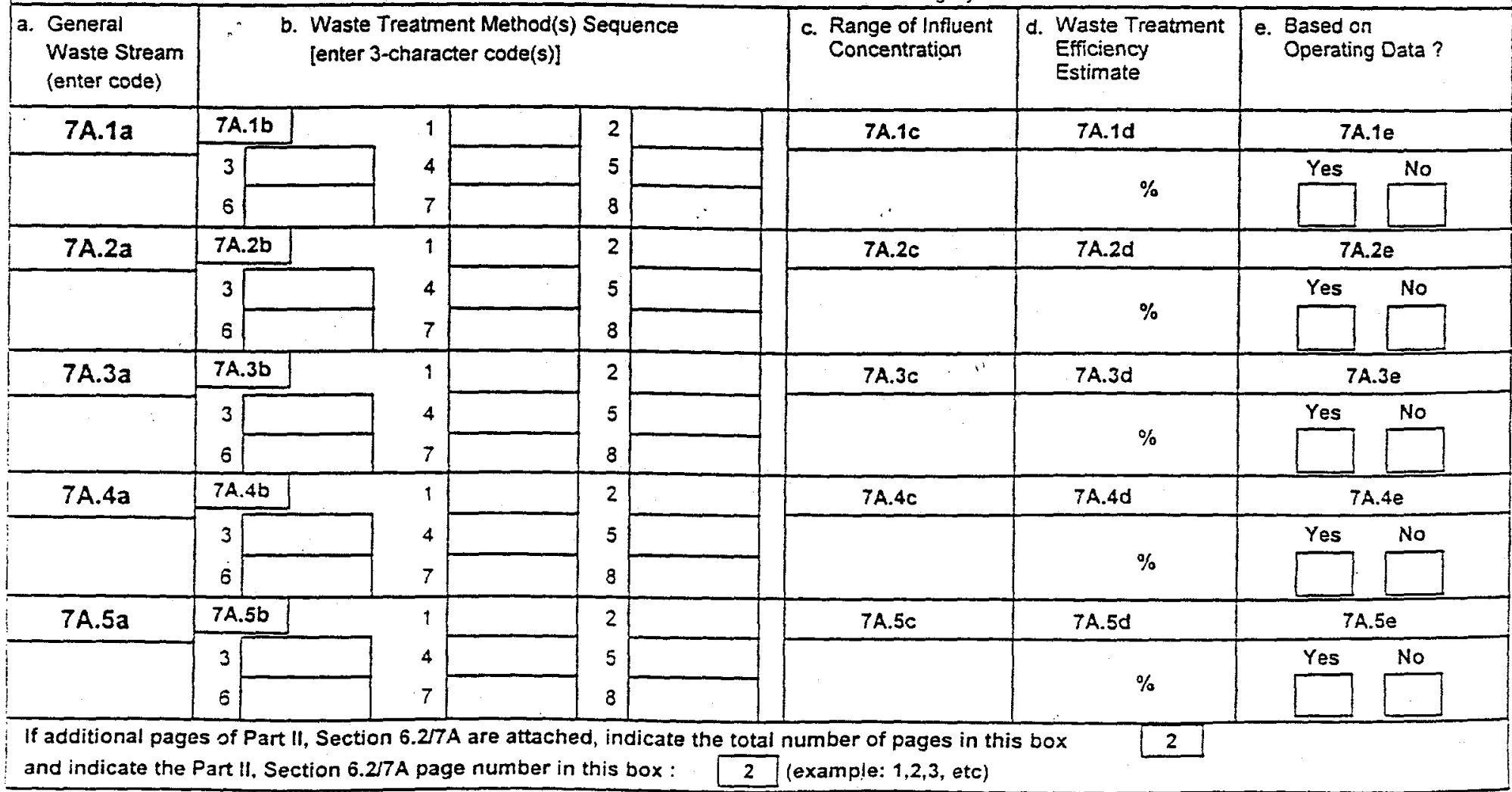




\section{EPA FORM $R$}

PART II. CHEMICAL-SPECIFIC INFORMATION. (CONTINUED)

\section{SECTION 7B. ON-SITE ENERGY RECOVERY PROCESSES}

$x$ Not Applicable (NA) - Check here if no on-site energy recovery is applied to any waste stream containing the toxic chemical or chemical category.

Energy Recovery Methods [enter 3-character code(s)]

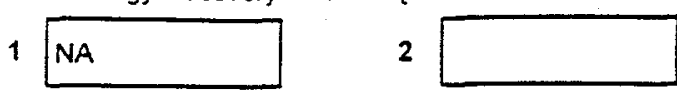

3

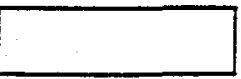

4

\section{SECTION 7C. ON-SITE RECYCLING PROCESSES}

Recyciing Methods [enter 3-character code(s)]

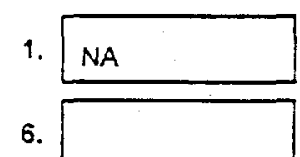

6.

2.

7.

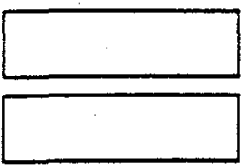

3.

8.

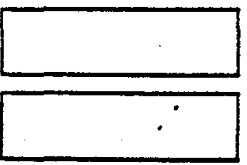

4.

9.

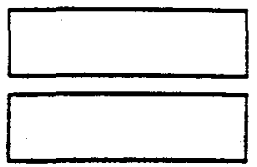

5.

10.
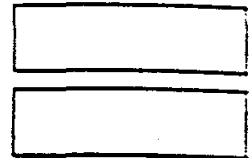

SECTION 8. SOURCE REDUCTION AND RECYCLING ACTIVITIES

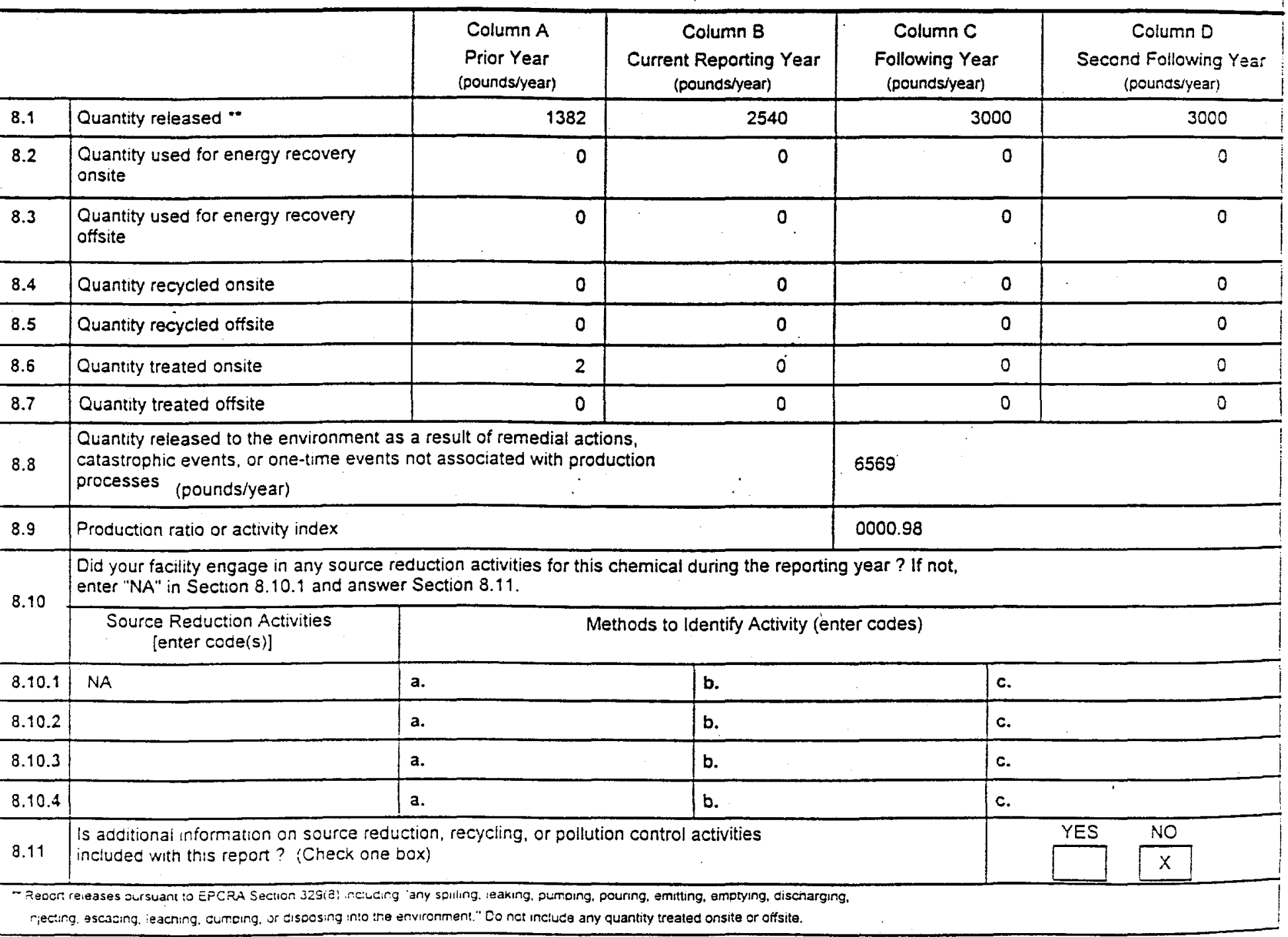

